\title{
Biomarcadores coronarios y evolución clínica alejada en pacientes con síndromes coronarios agudos sin elevación del segmento ST
}

\author{
Ricardo Baeza $\mathbf{V}^{1}$, Ramón Corbalán $\mathrm{H}^{1}$, Pablo Castro $\mathbf{G}^{1}$, \\ Mónica Acevedo B ${ }^{1}$, Teresa Q uiroga $\mathbf{G}^{2}$, Paola Viviani $\mathrm{G}^{3}$. \\ Coronary biomarkers and long-term \\ clinical outcome in acute coronary \\ syndrome without ST segment elevation
}

Background: The use of new biomarkers improved risk stratification for patients with acute coronary syndromes (ACS). Aim: To evaluate the relationship between multiple biomarkers and long-term clinical outcome in ACS without ST segment elevation. Patients and Methods: Consecutive patients presenting with suspected ACS were studied. On admission to the emergency room, serum was obtained to determine highly sensitive $\mathrm{C}$ reactive protein (hsCRP), erythrocyte sedimentation rate (ESR), lipoprotein (a) (LPa) and soluble P selectin (sPS). Clinical endpoints were mortality and a composite endpoint of major adverse cardiovascular events (MACE) including death, re-infarction, and angina. Results: Seventy patients, aged $63 \pm 13$ years, 54 males, were studied. Final diagnosis was unstable angina in $71 \%$ and non-ST-segment elevation myocardial infarction in 29\%. MACE and mortality rate were $17 \%$ and $5.8 \%$, respectively. We found higher plasma levels of hsCRP, ESR and Lp(a) in patients with MACE ( $p=0.032, p=0.015$ and $p=0.010$, respectively). Plasma levels of hsCRP and ESR were also higher in patients who died during the follow up ( $\mathrm{p}=0.002$ y $\mathrm{p}=0.045$, respectively). Conclusion: Plasma levels of inflammatory markers and atherosclerosis biomarkers are associated with a worse long-term clinical outcome in ACS without ST segment elevation. The inclusion of these biomarkers in the routine blood test on admission, could improve risk stratification of patients with ACS in the future (Rev Méd Chile 2005; 133: 1275-93).

(Key Words: Angina, unstable; C-reactive protein; Coronary arteriosclerosis; Lipoprotein (a); P-selectin)

Recibido el 16 de noviembre, 2004. Aceptado el 7 de junio, 2005.

Estudio financiado por la Sociedad Chilena de Cardiología y Cirugía Cardiovascular: Grant al Investigador Joven.

Departamento de Enfermedades Cardiovasculares ${ }^{1}$, Laboratorio Clínico ${ }^{2}$ y Departamento de Salud Pública ${ }^{3}$, Pontificia Universidad Católica de Chile.

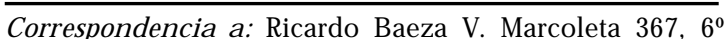

Piso, Unidad Coronaria. Santiago, Chile. Teléfono: 562-

3543624. Fax: 562-6392037. E mail: rbaeza@puc.cl 
os síndromes coronarios agudos (SCA), se Loriginan a partir de la rotura o erosión de una placa arteriosclerótica vulnerable, lo que es seguido de un proceso de activación y agregación plaquetaria y transformación celular ${ }^{1}$. Lo anterior puede llevar a la formación de un trombo oclusivo en las arterias y favorecer espasmo coronario, con la consecuente isquemia miocárdica ${ }^{2}$. Durante este accidente de placa, se activan una serie de mediadores, tanto humorales como celulares que pueden ser medidos y utilizados como biomarcadores en este proceso.

Diversos biomarcadores han sido relacionados con algunos de los procesos involucrados en la génesis de los SCA. Sin embargo, su utilidad en la práctica clínica no siempre es clara ni práctica. Dentro de las características ideales que debe tener un biomarcador, están la capacidad de reflejar la magnitud del proceso medido, modificarse por efecto de las terapias, tener una metodología bien definida para su determinación y entregar información diagnóstica y pronóstica que pueda ser usada en la estratificación de riesgo de pacientes con SCA.

La estratificación de riesgo de los pacientes con SCA se ha basado, generalmente, en variables clínicas y demográficas que pueden ser adquiridas al momento del ingreso de un paciente al servicio de urgencia. El adicionar biomarcadores a estas escalas tiene como atractivo la potencial optimización de la estratificación de riesgo. Esto permitiría, por un lado, identificar aquellos pacientes que se benefician de un tratamiento más agresivo y, por otro, evitar el empleo de exámenes invasivos en pacientes de bajo riesgo. Sin embargo, antes de incorporar un biomarcador, debemos estudiar su asociación con la evolución clínica de la enfermedad y la factibilidad de ser usados en ambientes como los servicios de urgencia o unidades de dolor torácico ${ }^{3-5}$.

El presente trabajo tiene por objetivo estudiar en forma prospectiva la relación entre biomarcadores de inflamación, aterosclerosis y activación plaquetaria, con la evolución clínica alejada de pacientes con SCA sin elevación del segmento ST, como un primer paso para el desarrollo de nuevas escalas de riesgo en este tipo de pacientes.

\section{MATERIAL Y MÉTODOS}

Selección y seguimiento de pacientes. Se incluyeron en este estudio a pacientes que consultaron en el servicio de urgencia del Hospital Clínico de la Universidad Católica por historia de dolor torácico sugerente de un SCA con o sin alteraciones electrocardiográficas, entre los meses de agosto y septiembre del año 2002.

Se excluyeron aquellos pacientes con elevación persistente del segmento ST, necesidad de estudio coronariográfico de urgencia, antecedentes de angioplastia o cirugía coronaria en los seis meses previos al ingreso, uso crónico de esteroides, disfunción renal conocida con creatinina plasmática $>2,0 \mathrm{mg} / \mathrm{dl}$, presencia de patologías oncológicas, inflamatorias o infecciosas y antecedente de ingesta crónica de niacina y estrógenos.

Todos los pacientes fueron ingresados a la Unidad de Cuidados Coronarios y tratados como SCA sin elevación del segmento ST. La terapia médica incluyó nitratos, heparina, betabloqueadores, aspirina e inhibidores de la glicoproteína IIb/ IIIa.

Cada paciente incluido en el estudio fue incorporado a una base de datos, donde se registraron los antecedentes demográficos, patologías asociadas y factores de riesgo cardiovascular. La estratificación se realizó en base a la escala de riesgo de TIMI (TRS) ${ }^{6}$.

El diagnóstico de infarto se basó en valores de CK y CKMB dos o más veces superior al límite máximo permitido por el laboratorio, $195 \mathrm{UI} / \mathrm{L}$ y $25 \mathrm{UI} / \mathrm{L}$, respectivamente.

El seguimiento clínico fue realizado cada 6 meses, hasta completar un año tras el evento índice y se efectuó en base al contacto telefónico y revisión de la ficha clínica de cada paciente.

Laboratorio. Se recolectaron muestras de sangre por venopunción directa en cada paciente. Las muestras fueron procesadas y analizadas en el laboratorio en forma independiente y sin acceso a la información clínica.

Los biomarcadores considerados específicamente para el presente estudio fueron velocidad de eritrosedimentación (VHS), proteína $\mathrm{C}$ reactiva ultrasensible (PCRus), lipoproteína (a) (Lp(a)) 
y P selectina plasmática soluble (PSs). Para el análisis sólo fueron considerados los valores plasmáticos obtenidos al momento de ingreso de los pacientes, previo a cualquier intervención 0 tratamiento.

Los niveles plasmáticos de PCRus y Lp(a) fueron determinados por técnica de nefelometría, nefelómetro BN 100 (Dade Behring). Los valores de VHS se determinaron por método clásico de Westergren. Los niveles de PSs se midieron por técnica de inmunoensayo (BBE6 R\&D Systems). Los valores considerados normales para cada biomarcador fueron: PCRus hasta $3 \mathrm{mg} / \mathrm{L}$; VHS hasta $10 \mathrm{~mm} / \mathrm{h}$; Lp(a) hasta $30 \mathrm{mg} / \mathrm{dl}$ y PSs hasta $40 \mathrm{ng} / \mathrm{ml}$.

En cada paciente se realizaron, además, exámenes convencionales consistentes en hemograma, creatinina, colesterol total, LDL, triglicéridos, perfil bioquímico, determinación de enzimas cardíacas convencionales creatinkinasa (CK), su fracción $\mathrm{MB}(\mathrm{CKMB})$ y ECG de 12 derivaciones.

Eventos clínicos. Los eventos clínicos evaluados fueron mortalidad por causa cardiovascular y nuevos eventos cardiovasculares (ECV), que incluyeron muerte, reinfarto, nuevos episodios de angina, necesidad de revascularización y hospitalización por nuevo SCA.

Estadística. Se utilizó test de correlación de Pearson para evaluar la relación entre cada uno de los diferentes marcadores. La comparación entre los valores de cada biomarcador según los eventos clínicos se realizó con test no paramétrico (Mann Whitney) para variables continuas no gaussianas. El análisis multivariado se realizó por regresión logística binaria y consideró sólo los biomarcadores en estudio y la evolución clínica alejada, muerte o nuevo evento cardiovascular, como variables dicotómicas. Todos los análisis estadísticos se realizaron con el programa MINI$\mathrm{TAB}^{\circledR}$ considerando un error $\alpha=0,05$.

\section{RESULTADOS}

Se incluyeron originalmente 80 pacientes, de los cuales se excluyeron 3 por necesidad de angio- plastia de urgencia, 4 pacientes por tener patologías extracardíacas que explicaban sus síntomas y 3 por tener creatinina mayor a 2,0 mg/dL. De esta manera, el análisis final se realizó en 70 pacientes.

El seguimiento clínico se completó en 100\% de los pacientes. Las características de nuestra población en estudio y los valores para cada biomarcador se muestran en la Tabla 1. Del total de la población en estudio, 71\% de los pacientes correspondió a pacientes con angina inestable y $29 \%$ a pacientes con infarto miocárdico sin elevación del segmento ST. El tiempo promedio de consulta desde el inicio de los síntomas fue de $2,8 \pm 2 \mathrm{~h}$.

Durante el periodo de seguimiento, 4 (5,7\%) pacientes (pts.) fallecieron por causa cardiovascular (uno de ellos por rotura ventricular) y 12 pts. (17\%) presentaron nuevos ECV, que se desglosan como sigue: 4 muertes, 2 con episodios de angina que requirieron ajuste de terapia, 3 con angina que requirió hospitalización o reintervenciones y 3 con nuevo infarto del miocardio.

Los valores para cada uno de los biomarcadores en estudio se muestran en la Tabla 1. Los factores de riesgo como sexo, edad, hipertensión, diabetes, tabaquismo y dislipidemia no se relacionaron significativamente con los valores plasmáticos de cada biomarcador.

El estudio de correlación entre los biomancadores mostró un coeficiente de 0,33 ( $p<0,05$ ) entre los valores de VHS y PCRus. El análisis entre el resto de los marcadores no mostró significancia estadística en esta serie.

El análisis univariado para la asociación entre los biomarcadores y la evolución clínica se muestra en las Figuras 1-4. La presencia de nuevos ECV durante el periodo de seguimiento se asoció con los niveles de PCRus $(25 \mathrm{mg} / \mathrm{L} \mathrm{v} / \mathrm{s} 6,5 \mathrm{mg} / \mathrm{L}$, $\mathrm{p}=0,032)$, de VHS $(18 \mathrm{~mm} / \mathrm{h} \mathrm{v} / \mathrm{s} 12 \mathrm{~mm} / \mathrm{h}, \mathrm{p}=0,015$ y de Lp(a) 18,6 mg/dL v/s 9,6 mg/dL, p=0,010. Los pacientes que fallecieron durante el seguimiento presentaron niveles significativamente más altos de PCRus (134 mg/L v/s 7,2 mg/L, $\mathrm{p}=0,002)$ y VHS (29 $\mathrm{mm} / \mathrm{h}$ v/s $12 \mathrm{~mm} / \mathrm{h}, \mathrm{p}=0,045)$. Los niveles de Lp(a) también fueron más elevados en los pacientes que fallecieron, pero sin alcanzar significancia estadística $(21 \mathrm{mg} / \mathrm{dL}$ v/s $10 \mathrm{mg} / \mathrm{dL}, \mathrm{p}=0,083)$. Los niveles plasmáticos de PSs no mostraron ninguna asociación significativa en relación a la evolución clínica. 
El análisis multivariado entre biomarcadores y la presencia de nuevos ECV se muestra en la Tabla 2. Este análisis demuestra que sólo los valores plasmáticos de $\mathrm{Lp}(\mathrm{a})$ fueron predictores de la evolución clínica, con $4 \%$ de aumento en la incidencia de nuevos ECV por cada incremento de unidad en el biomarcador. Los valores de PCRus, aunque mostraron una tendencia en la predicción de ECV, no alcanzaron significancia estadística en esta serie.

El análisis multivariado entre biomarcadores y mortalidad, se muestra en la Tabla 3. Sólo los valores de PCRus mostraron ser predictores en el seguimiento alejado, con 3\% de aumento en la mortalidad por cada incremento de unidad de este biomarcador.

Tabla 1. Características clínicas de los pacientes y valores de los biomarcadores

Edad, años \pm DS

Hombres, n (\%)

Diabetes, $\mathrm{n}(\%)$

Hipertensión, n (\%)

Dislipidemia, n (\%)

Tabaquismo activo, n (\%)

Infarto/angina previa, $\mathrm{n}(\%)$

CRM previa, n (\%)

APC previa, n (\%)

Diagnóstico final

Angina inestable, n (\%)

Infarto, n (\%)

ECG ingreso

Normal, n (\%)

Isquemia, $\mathrm{n}(\%)$

No interpretable, n (\%)

Tratamiento previo

Aspirina, n (\%)

Clopidogrel, n (\%)

Betabloqueadores, n (\%)

IECA, n (\%)

Estatinas, $\mathrm{n}(\%)$

Escala de riesgo/TRS

$<3, \mathrm{n}(\%)$

3-4, n (\%)

$5-7, \mathrm{n}(\%)$

CK UI/L* 137 (72; 345)

$\mathrm{CK} / \mathrm{MB} \mathrm{UI} / \mathrm{L}^{*}$

PCRus mg/ $\mathrm{L}^{*}$

VHS $\mathrm{mm} / \mathrm{h}^{*}$

Lp(a) $\mathrm{mg} / \mathrm{dL}^{*}$

PSs $n g / \mathrm{ml}^{*}$
$63 \pm 13$

54

15

44

34

31

23

11

7

49

21

24

44

2

20

$19 \quad(15 ; 45)$

7,6 (3; 20)

$13 \quad(6 ; 30)$

$10 \quad(5 ; 21)$

$44 \quad(28 ; 62)$

$\mathrm{CRM}=$ cirugía revascularización miocárdica; $\mathrm{APC}=$ angioplastia coronaria; $\mathrm{IECA}=$ inhibidores de la enzima convertidora de angiotensina; TRS= TIMI risk score; $\mathrm{CK}=$ creatinkinasa; $\mathrm{CK}-\mathrm{MB}=$ fracción $\mathrm{MB}$; $\mathrm{PCRus=}$ proteína C reactiva ultrasensible; VHS= velocidad de eritrosedimentación; $L p(a)=$ lipoproteína $(a) ; P S s=P$ selectina plasmática soluble. "Valores de biomarcadores expresados como medianas más percentiles 25, 75. 


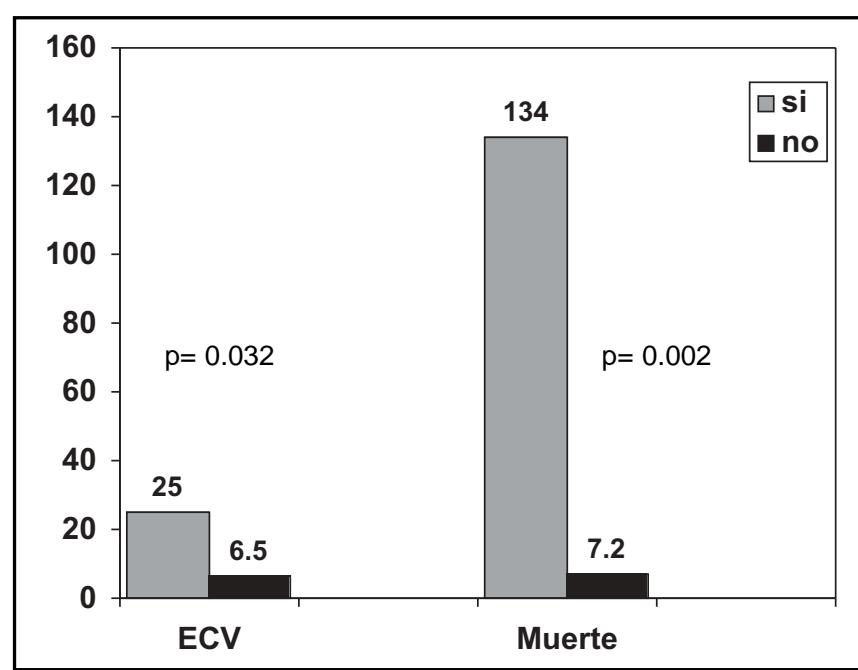

FiguRA 1. Relación entre los niveles plasmáticos de PCRus y evolución clínica alejada; PCRus= proteína $\mathrm{C}$ reactiva ultrasensible.
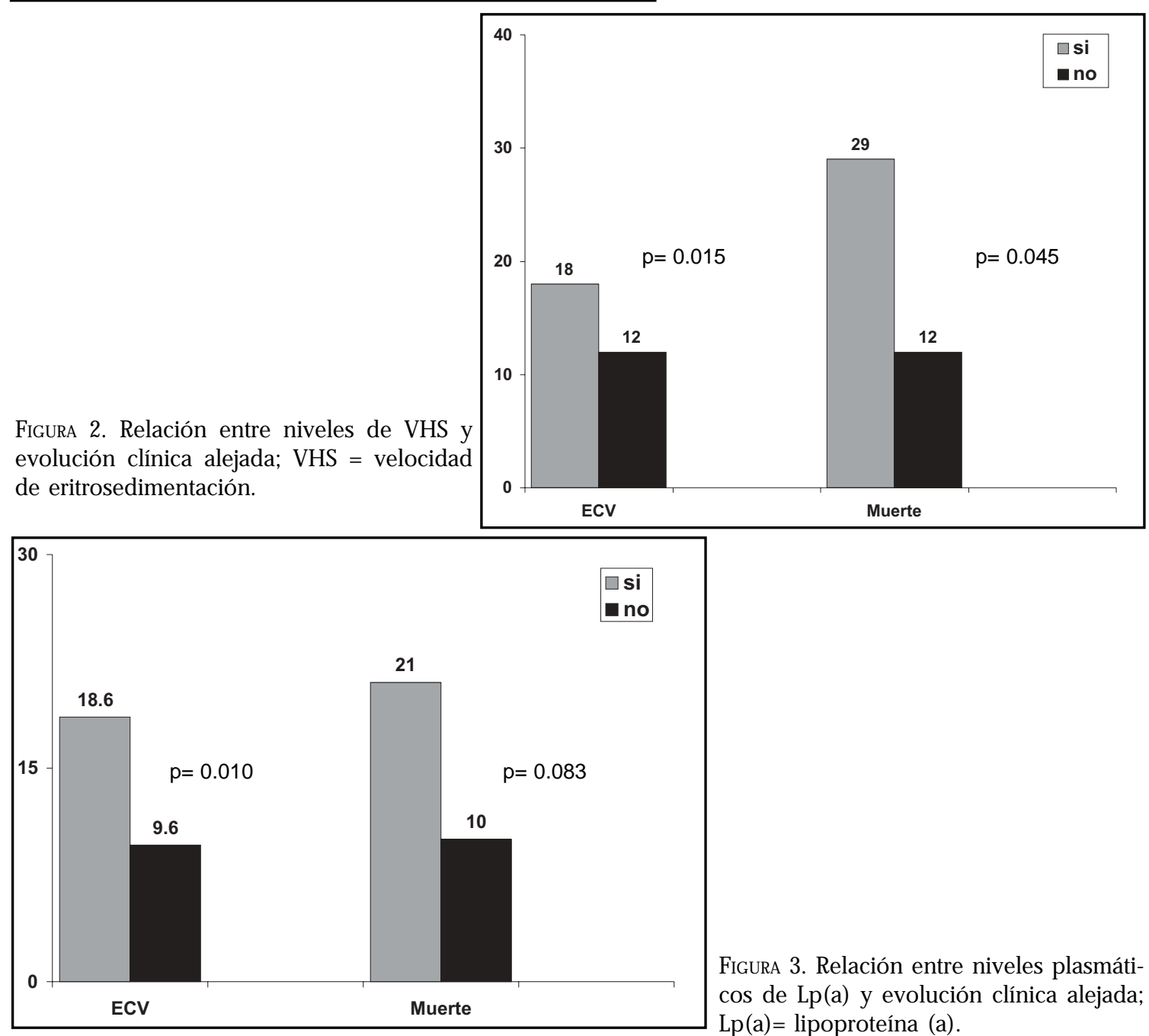

FIgURA 3. Relación entre niveles plasmáticos de Lp(a) y evolución clínica alejada; $\mathrm{Lp}(\mathrm{a})=$ lipoproteína $(\mathrm{a})$. 


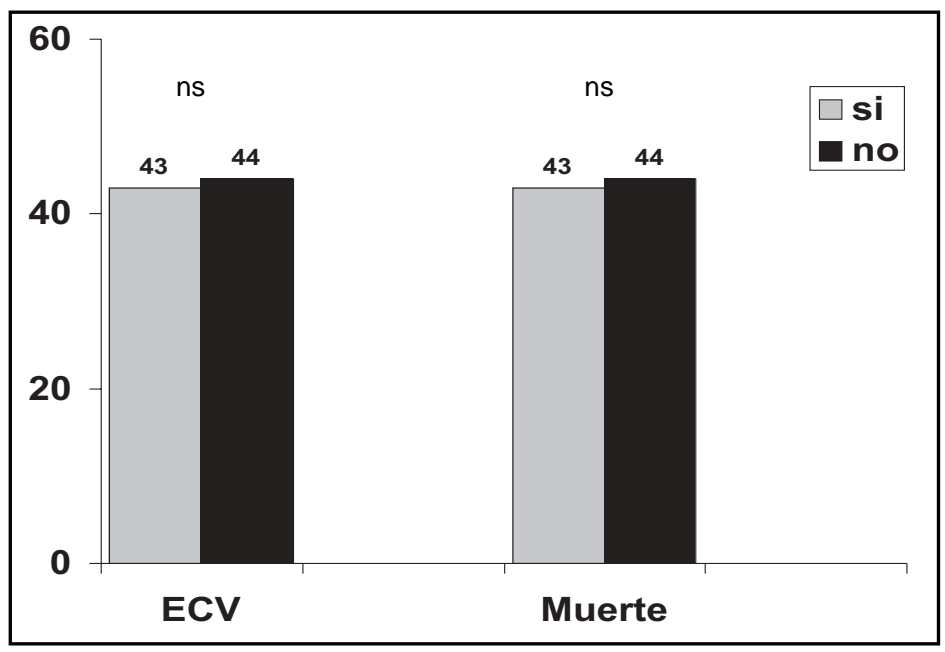

FigURA 4. Relación entre niveles plasmáticos de PSs y evolución clínica alejada; PSs $=$ P selectina plasmática soluble.

Tabla 2. Análisis multivariado para biomarcadores y nuevos eventos coronarios

\begin{tabular}{|lrrr|}
\hline & OR & IC $95 \%$ & Valor $p$ \\
\hline PCRus & 1,01 & $1,0-1,03$ & 0,069 \\
VHS & 1,01 & $0,98-1,04$ & 0,243 \\
Lpa & 1,04 & $1,01-1,08$ & $0,044^{*}$ \\
sPS & 1,01 & $0,9-1,03$ & 0,601 \\
\hline
\end{tabular}

PCRus= proteína $\mathrm{C}$ reactiva ultrasensible; Lp(a) lipoproteína a; VHS= velocidad de eritrosedimentación; PSs= P Selectina plasmática soluble. OR= odds ratio; IC= intervalo de confianza límites para 95\%.

Tabla 3. Análisis multivariado para biomarcadores y mortalidad

\begin{tabular}{|lrrr|}
\hline & OR & CI 95\% & Valor P \\
\hline PCRus & 1,03 & $1,01-1,5$ & $0,006^{*}$ \\
VHS & 0,99 & $0,98-1,06$ & 0,431 \\
Lpa & 1,0 & $0,95-1,06$ & 0,975 \\
PSs & 1,001 & $0,97-1,05$ & 0,614 \\
\hline
\end{tabular}

PCRus= proteína $\mathrm{C}$ reactiva ultrasensible; Lp(a) lipoproteína a; VHS= velocidad de eritrosedimentación; PSs= P Selectina plasmática soluble. OR= odds ratio; IC= intervalo de confianza límites para 95\%. 


\section{DisCUSIÓN}

Este estudio demuestra, por primera vez en nuestro medio, la relación entre biomarcadores coronarios de inflamación y aterosclerosis con la evolución clínica alejada en pacientes con SCA sin elevación del segmento ST. Este resultado es el primer paso en la validación de nuevos biomarcadores y la elaboración de nuevas y mejores escalas de riesgo en este tipo de pacientes.

La actividad inflamatoria en la placa aterosclerótica es un factor determinante en la génesis de los SCA y puede ser medida a través de varios biomarcadores. El más extensamente estudiado de estos biomarcadores es la PCRus ${ }^{7,8}$, que no siendo específica de los procesos inflamatorios coronarios, ha demostrado su utilidad en la estratificación de riesgo y en la predicción de nuevos eventos clínicos en pacientes con SCA ${ }^{9,10}$. Nuestros resultados demuestran que por cada aumento en unidad de los niveles de PCRus el riesgo de mortalidad aumenta en 3\%. Esto ratifica que este marcador, aun en casuísticas pequeñas, es útil en la predicción de eventos a largo plazo, así como lo han demostrado experiencias clínicas con un mayor número de pacientes ${ }^{10}$.

La utilidad de la VHS en pacientes con SCA ha sido previamente reportada ${ }^{11,12}$. Al igual que la PCRus, los valores de VHS se relacionan con la magnitud del fenómeno inflamatorio, teniendo una potencial utilidad en la estratificación de este tipo de pacientes. En nuestro estudio, los valores de PCRus y VHS se relacionaron significativamente entre sí y fueron más elevados en aquellos pacientes con una evolución clínica adversa, lo cual ha sido demostrado en otras experiencias ${ }^{13}$. Uno de los atractivos de este marcador, es que se trata de un examen ampliamente difundido y de bajo costo, que potencialmente nos permitiría mejorar la estratificación de riesgo. Sin embargo, su falta de especificidad hace que su uso aislado deba ser tomado con cautela.

El proceso aterosclerótico, influido por factores genéticos y ambientales, juega un rol preponderante en la actividad de la placa ${ }^{14}$. En la práctica clínica, ante la ausencia de un marcador exclusivo de la actividad aterosclerótica, lo más usado es el control de los lípidos y lipoproteínas. La lipoproteína (a) está compuesta de dos diferentes subuni- dades (apo A y apo B) y tiene características químicas y estructurales similares a las de las LDL, atribuyéndosele propiedades protrombóticas y antifibrinolíticas ${ }^{15}$. Su asociación con la evolución de la enfermedad coronaria a largo plazo ha sido demostrada en estudios clínicos, pero su valor pronóstico no ha sido validado en forma consistente en la literatura, en especial en pacientes con $\mathrm{SCA}^{15,16}$. Los resultados obtenidos en nuestra serie muestran una clara asociación entre los niveles de este marcador y la evolución clínica alejada, así, por cada unidad que aumenta los niveles de Lp(a) el riesgo de un nuevo ECV aumenta en 4\%. Este hallazgo permite pensar en su potencial utilidad para estratificar riesgo en pacientes con SCA y en futuros programas de prevención primaria o secundaria.

La P selectina, es una proteína que forma parte de las moléculas de adhesión que se expresan en la superficie de la plaqueta y está estrechamente ligada a los procesos de agregación. Existen dos formas medibles, la plaquetaria, que es aquella fracción unida a la superficie de la plaqueta y la soluble, que corresponde a la porción libre en el plasma. Experiencias clínicas han demostrado que pacientes con SCA tienen niveles más altos de PS, tanto plaquetaria como soluble, en comparación con una población control ${ }^{17-19}$, y que incluso sería útil en la diferenciación del dolor torácico en los servicios de urgencia ${ }^{19}$. En nuestra experiencia, encontramos que el valor promedio de los niveles de PS fue más alto que el conocido para la población normal, sin embargo no demostró estar asociado a la evolución clínica. Una probable explicación para este hecho es la eventual influencia de los diferentes métodos de medición usa$\operatorname{dos}^{20}$, y el uso de una sola determinación en vez de muestras seriadas, diferencias que pueda haber entre las fracciones solubles y plaquetarias de dicho marcador ${ }^{21,22}$ o las características de la población estudiada. En nuestro estudio la decisión de usar PSs fue guiada por sus ventajas técnicas en comparación con la determinación de las PSp, lo cual la hace más apropiada en el contexto de los Servicios de Urgencia y Unidades de Dolor Torácico 3,4 .

Es importante destacar que por lo complejo del proceso y dado el grado de múltiples interacciones que se dan a nivel molecular, es a nuestro 
juicio, imposible contar con marcadores "puros" para un determinado proceso. Así por ejemplo, Lp(a) tendría además efectos protrombogénicos y P-Selectina es mediador de algunas etapas de la inflamación en forma indirecta ${ }^{18}$. Esto debe tomarse en cuenta a la hora de elegir un biomarcador "ideal" o en la elección de un biomarcador sustituto, en los cuales la relación de un determinado biomarcador con el proceso biológico en estudio es necesaria pero no suficiente para explicar la evolución clínica de los pacientes ${ }^{23}$.

Dentro de las limitaciones de nuestro trabajo destaca el pequeño número de pacientes incluidos, lo cual nos impide realizar un análisis estadístico multivariado incorporando otros factores (ej: edad, sexo, efecto de terapias, etc.) Sin embargo ésta es la serie más numerosa que ha estudiado estos marcadores en nuestro medio. El uso de una sola determinación para cada biomarcador, en vez de muestras secuenciales, podría estar subestimando la eventual utilidad de ciertos biomarcadores (ej:

\section{REFERENCIAS}

1. Fuster V, Badimon J, Chesebro JH, Falon JT. Plaque rupture, thrombosis, and therapeutic implications. Haemostasis 1996; 26 Suppl 4: 269-84.

2. Ross R. Atherosclerosis; an inflammatory disease. N Eng J Med 1999; 340: 115-26.

3. Isa $R$, Castro P, Pérez $O$, Garayar B, Baeza $R$, Vergara I et al. Hacia el desarrollo de una unidad de dolor torácico. Estudio Piloto. Rev Chil Cardio 2003; 22: 123.

4. Casagranda I, Boverio R, Baio R, Ceeconi D, Marenco M. Chest pain unit and decentralized testing of cardiac markers. Clin Chim Acta 2001; 311: 63-6.

5. Storrow AB, Gibler WB. The role of cardiac markers in the emergency department. Clin Chim Acta 1999; 284: 187-96.

6. Antman EM, Cohen M, Bernink PJ, McCabe CH, Horacek T, Papuchis G et al. The TIMI risk score for unstable angina/non ST elevation MI: A method for prognostication and therapeutic decision making. JAMA 2000; 284: 835-42.

7. Heeschen C, Hamm CW, Bruemmer J, Simoons ML. Predictive value of $\mathrm{C}$ reactive protein and Tropo-
PSs). Sin embargo debemos tener en cuenta que muchos de estos marcadores son susceptibles de modificarse no sólo por la evolución natural de la enfermedad, sino también por intervenciones usadas en la práctica clínica tales como angioplastia, cirugía, drogas antiplaquetarias, etc. De ahí la potencial ventaja de la determinación única en la sala de urgencia, antes de que cualquier otro procedimiento se haya realizado. Las características de nuestra población en estudio, joven y de relativo bajo riesgo, también puede haber influido en nuestros hallazgos.

En conclusión, los niveles plasmáticos de biomarcadores de actividad inflamatoria y de aterosclerosis se asocian con la evolución clínica alejada en pacientes con SCA sin elevación del segmento ST. Su eventual utilidad para la estratificación de riesgo y selección de terapias en pacientes con SCA, así como la relación con otros biomarcadores coronarios debe ser explorada en mayor escala.

nin $\mathrm{T}$ in patients with unstable angina: a comparative analysis: CAPTURE Investigators. J Am Coll Cardiol 2000; 35: 1535-42.

8. Mazzone A, De Servi S, Ricevuti G, Mazzucchem I, Fossati G, PasotTi D et al. Increased expression of neutrophil and monocyte adhesion molecules in unstable coronary artery disease. Circulation 1993; 88: 358-63.

9. MulvihiL N, Foley JB, Murphy R, Crean P, Walsh M. Evidence of prolonged inflammation in unstable angina and non Q wave myocardial infarction. J Am Coll Cardiol 2000; 36: 1210-5.

10. Biasucci LM, Luzzo G, Grilo RL, Calugiuri G, Rebuzzi AG, Buffon A ET aL. Elevated levels of $C$ reactive protein at discharge in patients with unstable angina predict recurrent instability. Circulation 1999; 99: 855-60.

11. Natali A, L'Abbate A, FerRannini E. Erythrocyte sedimentation rate, coronary atherosclerosis and cardiac mortality. Eur Heart J 2003, 24: 639-48.

12. EriksSen $G$, Liestol $K$, Bjornholt JV, Stormorken $H$, Thaulow E, ERIKSSEN J. Erythrocyte sedimentation rate: a possible marker of atherosclerosis and a strong predictor of coronary heart disease mortality. Eur Heart J 2000; 21: 1614-20. 
13. Bholasingh R, Cornel JH, Kamp O, van Straaien JP, SANDERS GT, DiJKSMAN L ET AL. The prognostic value of markers of inflammation in patients with troponin T-negative chest pain before discharge from the emergency department. Am J Med 2003; 115: 521-8.

14. DaVIES MJ. Stability and instability: two faces of coronary atherosclerosis. Circulation 1996; 94: 2013-20.

15. Marcovina SM, Koschinsky ML. Lipoprotein (a) as a risk factor for coronary artery disease. Am J Cardiol 1998; 82: 57-66.

16. Igarashi Y, Aizana Y, Satoh T, Konno T, Ojima K, Aizawa Y. Predictors of adverse long-term outcome in acute myocardial infarction patients undergoing primary percutaneous transluminal coronary angioplasty: with special reference to the admission concentration of lipoprotein (a). Circ J 2003; 67: 605-11.

17. Gawas M, Reininger A, Neumann FJ. Platelet Function and Platelet-Leukocyte Adhesion in Symptomatic Coronary Heart Disease. Effects of Intravenous Magnesium. Thromb Res 1996; 83: 341-9.

18. Ikeda $H$, TaKajo $Y$, IchiKi $K$, Ueno T, MaKi S, Noda T ET AL. Increased soluble form of $P$ Selectin in patients with unstable angina. Circulation 1995; 92: 1693-6.

19. Hollander J, Muttreja R, Dalesandro M, Shofer F. Risk stratification of emergency department patients with acute coronary syndromes using $\mathrm{P}$ selectin. J Am Coll Cardiol 1999; 34: 95-105.

20. Sarma J, Laan C, Alam S, Jha A, Fox K, Dransfield I. Increased platelet binding to circulating monocytes in acute coronary syndromes. Circulation 2002; 105: 2166-71.

21. Serebruany VL, Kereiakes DJ, Dalesandro MR, GurBEL PA. The flowcitometer model markedly affects measurements of ex vivo whole blood plateletbound $\mathrm{P}$ selectin expresion in patients with chest pain: are we comparing apples with oranges? Thromb Res 1999; 96: 52-6.

22. Gurbel PA, Kereiakes DJ, Serebruany VL. Soluble $P$ selectin is not a surrogate marker for platelet $P$ selectin, evidence from a multicenter chest pain study group. J Thromb Thrombolysis 2000; 10: 1522.

23. Fieming TR, DeMETS DL Surrogate end points in clinical trials: are we being misled? Ann Intern Med 1996; 125: 605-13. 